\title{
Core-Halo Distribution in the Hamiltonian Mean-Field Model
}

\author{
Renato Pakter and Yan Levin \\ Instituto de Física, Universidade Federal do Rio Grande do Sul, Caixa Postal 15051, CEP 91501-970, \\ Porto Alegre, Rio Grande do Sul, Brazil
}

(Received 29 November 2010; revised manuscript received 19 April 2011; published 20 May 2011)

\begin{abstract}
We study a paradigmatic system with long-range interactions: the Hamiltonian mean-field (HMF) model. It is shown that in the thermodynamic limit this model does not relax to the usual equilibrium Maxwell-Boltzmann distribution. Instead, the final stationary state has a peculiar core-halo structure. In the thermodynamic limit, HMF is neither ergodic nor mixing. Nevertheless, we find that using dynamical properties of Hamiltonian systems it is possible to quantitatively predict both the spin distribution and the velocity distribution functions in the final stationary state, without any adjustable parameters. We also show that HMF undergoes a nonequilibrium first-order phase transition between paramagnetic and ferromagnetic states.
\end{abstract}

DOI: 10.1103/PhysRevLett.106.200603

PACS numbers: 05.20. $-\mathrm{y}, 05.45 .-\mathrm{a}, 05.70 . \mathrm{Ln}$

Since the early work of Clausius, Boltzmann, and Gibbs it has been known that for particles interacting through short-range potentials, the final stationary state reached by a system corresponds to the thermodynamic equilibrium [1]. Although no exact proof exists, in practice it is found that nonintegrable systems with a fixed energy and number of particles (microcanonical ensemble) always relax to a unique stationary state which only depends on the global conserved quantities: energy, momentum, and angular momentum. The equilibrium state does not depend on the specifics of the initial particle distribution. The situation is very different for systems in which particles interact through long-ranged unscreened potentials. This is the case for gravitational systems and confined one component plasmas [2,3]. For these systems, in the thermodynamic limit, the collision duration time diverges, and the thermodynamic equilibrium is never reached [4]. Instead, as time $t \rightarrow \infty$, these systems become trapped in a stationary state characterized by a broken ergodicity [5-7]. Unlike the thermodynamic equilibrium, the stationary state depends explicitly on the initial particle distribution. Over the last 50 years, there has been a great effort to predict the final stationary state without having to explicitly solve the $N$-body dynamics or the collisionless Boltzmann (Vlasov) equation. Qualitatively, it has been observed that for many different systems the nonequilibrium stationary state has a peculiar core-halo shape. Recently, an ansatz solution to the Vlasov equation has been proposed which allowed us to explicitly calculate the core-halo distribution function for confined plasmas and self-gravitating systems $[2,3]$. In this Letter we will show that an ansatz solution is also possible for the Hamiltonian mean-field (HMF) model. The theory proposed allows us also to locate the nonequilibrium para-to-ferromagnetic phase transition, which earlier theories incorrectly predicted to be of second order [8]. All of the results are compared with the molecular dynamics simulations performed using a symplectic integrator, and are found to be in excellent agreement.

The HMF model consists of $N, X Y$ interacting spins, whose dynamics is governed by the Hamiltonian

$$
H=\sum_{i=1}^{N} \frac{p_{i}^{2}}{2}+\frac{1}{2 N} \sum_{i, j=1}^{N}\left[1-\cos \left(\theta_{i}-\theta_{j}\right)\right],
$$

where angle $\theta_{i}$ is the orientation of the $i$ th spin and $p_{i}$ is its conjugate momentum [8-10]. The macroscopic behavior of the system is characterized by the magnetization vector $\mathbf{M}=\left(M_{x}, M_{y}\right)$, where $M_{x} \equiv\langle\cos \theta\rangle, M_{y} \equiv\langle\sin \theta\rangle$, and $\langle\cdots\rangle$ stands for the average over all particles. The modulus, $M=|\mathbf{M}|$ serves as the order parameter which measures the coherence of the spin angular distribution: for $M=0$ we have a completely incoherent state, whereas for finite $M$ there is some degree of coherence. Hamilton's dynamic equations for each spin can be expressed in terms of the total magnetization and take a particularly simple form $\ddot{\theta}_{i}=F\left(\theta_{i}\right)$, where the force on each spin is $F\left(\theta_{i}\right)=$ $-M_{x} \sin \theta_{i}+M_{y} \cos \theta_{i}$. The average energy per particle is $u=H / N=\left\langle p^{2}\right\rangle / 2+\left(1-M^{2}\right) / 2$. Since the Hamiltonian does not explicitly depend on time, $u$ is conserved along the temporal evolution. For simplicity we will consider initial distributions of the "water-bag" form in the $(\theta, p)$ reduced phase space ( $\mu$ space). Without loss of generality, we choose a frame of reference where $\langle\theta\rangle=0$ and $\langle p\rangle=0$. The one-particle initial distribution function then reads

$$
f_{0}(\theta, p)=\frac{1}{4 \theta_{0} p_{0}} \Theta\left(\theta_{0}-|\theta|\right) \Theta\left(p_{0}-|p|\right)
$$

where $\Theta$ is the Heaviside step function, and $\theta_{0}$ and $p_{0}$ are the maximum absolute values of the angle and the momentum, respectively. Such initial distributions are characterized by $M_{x}=M_{0}, M_{y}=0$, and $u=p_{0}^{2} / 6+\left(1-M_{0}^{2}\right) / 2$, where $M_{0}=\sin \left(\theta_{0}\right) / \theta_{0}$ is the initial magnetization. Because of the symmetry of $f_{0}$ with respect to $\theta=0$, in 
the thermodynamic limit $M_{y}=0$ throughout the evolution, so that the macroscopic dynamics is completely determined by $M_{x}(t)$.

As the system evolves, the particle distribution in the phase space changes and eventually reaches a stationary state or a limit cycle. If $N$ is finite, the stationary state will be described by the equilibrium Maxwell-Boltzmann (MB) distribution. In the thermodynamic limit $N \rightarrow \infty$, however, the system becomes trapped in a nonergodic nonmixing state, the lifetime of which diverges with the number of particles. In this limit, the dynamical evolution of oneparticle distribution function $f(\theta, p, t)$ is governed exactly by the collisionless Boltzmann (Vlasov) equation [11],

$$
\frac{\partial f}{\partial t}+p \frac{\partial f}{\partial \theta}+F(\theta) \frac{\partial f}{\partial p}=0 .
$$

The left-hand side of this equation is just the convective derivative of the one-particle distribution function. Therefore, a collisionless Hamiltonian system evolves over the phase space as an incompressible fluid. Furthermore, the incompressibility implies that during the temporal evolution the phase-space density can never exceed the maximum of the initial distribution function.

Although the MB distribution is also a stationary solution of the Vlasov equation, unlike for the Boltzmann equation, it is not a global attractor of the dynamics, so that an arbitrary initial distribution will not evolve to the MB equilibrium. The collisionless relaxation described by the Vlasov equation for systems with long-range interactions is, therefore, much more complex than the collisional relaxation governed by the usual Boltzmann equation for systems with short-range forces.

The Vlasov equation is time reversible. Thus, on a finegrained scale, temporal evolution never ends. There is no fine-grained attractor for the Vlasov dynamics. In practice, however, one can never have infinite resolution, and there is a finite maximum precision that one can reach in any experiment or a numerical simulation. It is on this coarsegrained scale that it appears that the evolution has reached a steady state. Unlike for the Boltzmann equation, however, the stationary coarse-grained distribution function depends explicitly on the initial condition.

Recently it has been observed that for systems with long-range interactions, such as self-gravitating clusters and plasmas [2,3], the final stationary state has a peculiar core-halo structure. The mechanism of core-halo formation is very similar to the process of evaporative cooling. As the dynamics evolves, macroscopic propagating density waves are formed. Some particles enter in resonance with the macroscopic oscillations gaining a large amount of energy at the expense of the collective motion. This is similar to the mechanism of Landau damping well known in plasma physics [12]. Resonant particles can gain sufficient energy to reach high energy states, thus forming a diffuse halo. On the other hand, the loss of energy dampens the macroscopic oscillations, so that the leftover particles become condensed into the low energy states, resulting in a dense core. However, because of the incompressibility of Vlasov dynamics, the core cannot completely freezei.e., collapse to the minimum of the potential energy. Instead, the distribution function of the core particles progressively approaches the maximum phase-space density allowed by the initial distribution-all the low energy states become fully occupied by the core particles. Although the HMF model appears to be very different from either self-gravitating clusters or confined plasmas, we find that its dynamical evolution follows exactly the same scenario as described above.

In the case of the HMF, the oscillations of the magnetization $M$ play the role of collective oscillations which drive some spins to higher energy states, leading to a halo formation. The macroscopic oscillations of $M$ are significantly damped in one or two periods of oscillation. The extent of the halo is determined on the same time scale. As a consequence of the conservation of the total energy, the remaining spins populate lower and lower energy states, until all of them become fully occupied up to the maximum phase-space density $\eta_{0}=1 / 4 \theta_{0} p_{0}$. In the final stationary state, the core distribution function is the same as that of a fully degenerate Fermi gas of spin-degeneracy $\eta_{0}$. The core distribution extends up to the Fermi energy $\varepsilon_{F}$. The value of $\varepsilon_{F}$ is yet unknown, and must be determined selfconsistently. We propose an ansatz for the core-halo distribution that describes the final (coarse-grained) stationary state reached by the HMF model at the end of its dynamical evolution:

$$
f_{s}(\theta, p)=\eta_{0}\left[\Theta\left(\varepsilon_{F}-\varepsilon\right)+\chi \Theta\left(\varepsilon_{h}-\varepsilon\right) \Theta\left(\varepsilon-\varepsilon_{F}\right)\right],
$$

where $\varepsilon\left(\theta, p, M_{s}\right)=p^{2} / 2+1-M_{s} \cos \theta$ is the singlespin energy, $\chi$ is the ratio between the halo and the core phase-space densities, $M_{s}$ is the stationary value of magnetization, and $\varepsilon_{h}$ is the maximum energy of the halo spins. The energy $\varepsilon_{h}$ is determined from the short-time dynamics of spins driven by the oscillations of the magnetization. To estimate this value we need an independent equation that describes the dynamical evolution of magnetization. We proceed as follows: taking the second derivative of $M_{x}$ we obtain $\ddot{M}_{x}=M_{x}\left\langle\sin ^{2} \theta\right\rangle-\left\langle p^{2} \cos \theta\right\rangle$. This equation requires the knowledge of the temporal evolution of $\left\langle\sin ^{2} \theta\right\rangle$ and $\left\langle p^{2} \cos \theta\right\rangle$, which leads to an infinite hierarchy of equations. To truncate the hierarchy, we assume that for short times $\left\langle\sin ^{2} \theta\right\rangle=1 / 2$ and $\left\langle p^{2} \cos \theta\right\rangle=\left\langle p^{2}\right\rangle\langle\cos \theta\rangle=$ $\left(2 u-1+M_{x}^{2}\right) M_{x}$, where use has been made of the conservation of energy, together with the condition $M=M_{x}$. We then find a dynamical equation satisfied by the magnetization

$$
\ddot{M}_{x}=-M_{x}\left(2 u+M_{x}^{2}-\frac{3}{2}\right) .
$$

This equation can be integrated numerically to provide the temporal evolution of $M_{x}(t)$. Since Eq. (5) was derived 
neglecting the correlations between angles and momentums, its validity extends only to very short times. However, the maximum energy of the halo is also determined by the very short-time dynamics. Thus, Eq. (5), should be sufficient to give a reasonable estimate of the value of the maximum halo energy. We adopt the following procedure to determine $\varepsilon_{h}$. For a given initial distribution, we determine the maximum energy attained by a group of noninteracting test-spins that are launched with the initial conditions selected from the distribution function, Eq. (2). Their dynamical evolution is governed by $\ddot{\theta}_{i}=-M_{x}(t) \sin \theta_{i}$ with $M_{x}(t)$ determined by Eq. (5), with $M_{x}(0)=M_{0}$ and $\dot{M}_{x}(t)=0$. We solve this equation over a short time corresponding to two periods of oscillation of $M_{x}$. The $\varepsilon_{h}$, then, corresponds to the maximum energy obtained by any of the test-spins.
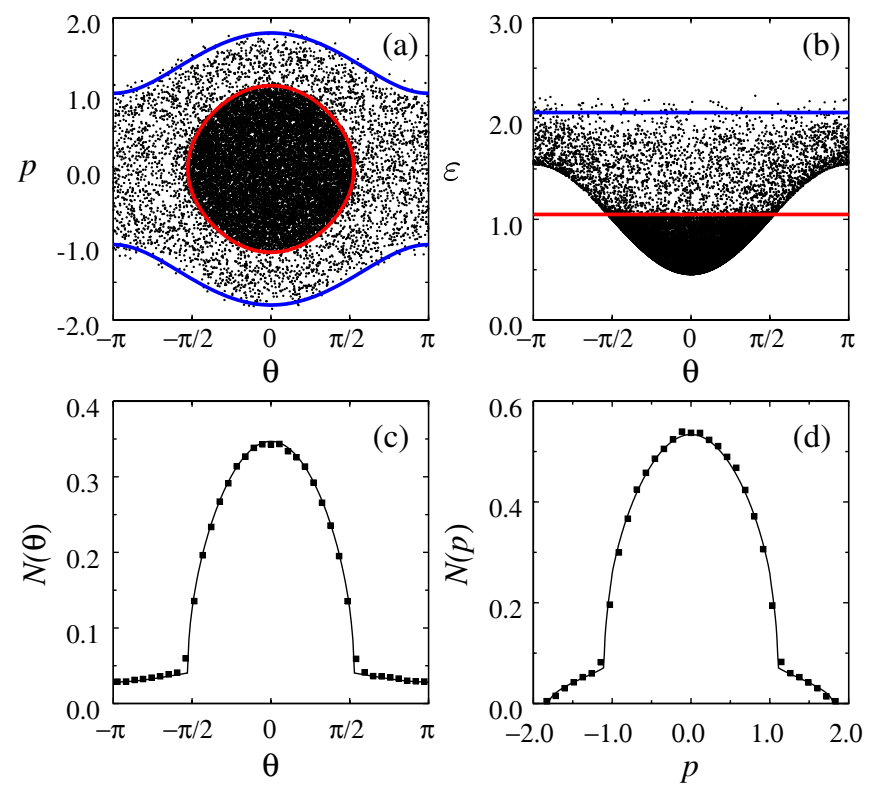

FIG. 1 (color online). Snapshots of (a) the phase space and (b) of spin energies $\varepsilon$, as a function of angle. The snapshot is taken at $t=10000$, using $N=20000$ spins. The solid curves correspond to the calculated Fermi energy $\varepsilon_{F}$ (red, inner circle) and to the maximum halo energy $\varepsilon_{h}$ (blue, outer curves), i.e., $\varepsilon\left(\theta, p, M_{s}\right)=p^{2} / 2+1-M_{s} \cos \theta=\varepsilon_{F}$ and $\varepsilon\left(\theta, p, M_{s}\right)=\varepsilon_{h}$, respectively. We see that the Fermi energy curve perfectly encloses the high density region. The maximum halo energy obtained using the test particle dynamics and Eq. (5) also delimits well the extent of the particle distribution in the phase space [blue upper line, panel (b)]. Panels (c) and (d) show the angle and the momentum distributions, respectively. Solid curves are the theoretical predictions obtained using the distribution function of Eq. (4), and points are the results of molecular dynamics simulations averaged over 20 runs. The initial distribution has $M_{0}=0.80$ and $u=0.55$. Note that the present theory predicts that the maximum energy attained by any spin will be bounded by $\varepsilon_{h}$, while theories based on generalized entropies predict that this energy distribution is unbounded, decaying either exponentially or algebraically [9], but see also [16].
Once $\varepsilon_{h}$ has been determined using the test particle dynamics, the remaining parameters of the final stationary distribution- $\varepsilon_{F}, \chi$, and $M_{s}$-are obtained by imposing the conditions of conservation of norm and of the total energy, as well as the closure equation for magnetization:

$$
\begin{gathered}
\int f_{s}(\theta, p) d \theta d p=1, \\
\int f_{s}(\theta, p) \varepsilon\left(\theta, p, M_{s}\right) d \theta d p=u, \\
\int f_{s}(\theta, p) \cos \theta d \theta d p=M_{s} .
\end{gathered}
$$

The equations above can be analytically evaluated in terms of elliptic integrals, forming a closed set of algebraic equations that must be solved numerically to determine $\varepsilon_{F}, \chi$, and $M_{s}$.

In Figs. 1(a) and 1(b) we show a snapshot of the phase space and of the distribution of spin energies. The corehalo separation can be seen very clearly. The energy $\varepsilon_{h}$ delimits the particle distribution over the phase space, while the Fermi energy restricts the extent of the core region. In this example the initial distribution has $M_{0}=0.80$ and $u=0.55$, while the magnetization in the

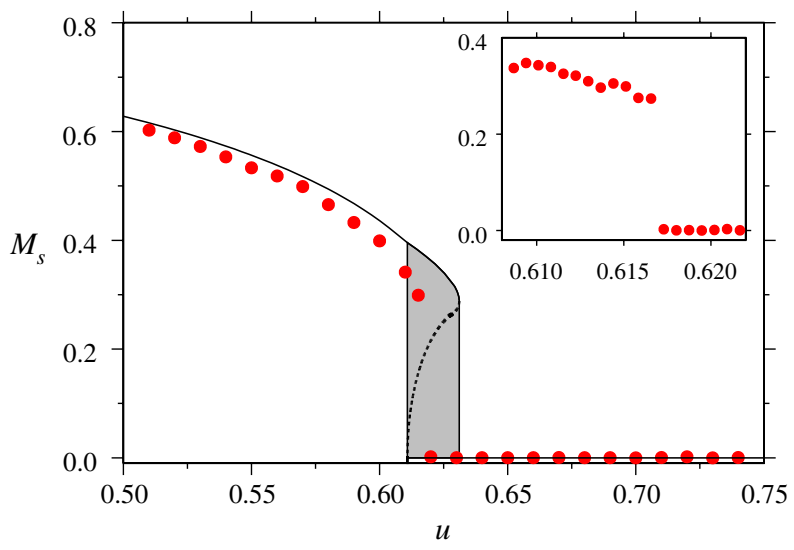

FIG. 2 (color online). Magnetization of the final stationary state $M_{s}$ as a function of the initial energy per particle $u$ for $M_{0}=0.40$. The curves are the theoretical predictions obtained using the distribution function of Eq. (4), and the points are the results of the molecular dynamics simulations with $N=10^{6}$ spins. The solid curve corresponds to the stable solutions, whereas the dashed curve corresponds to unstable solutions. The gray area corresponds to the metastable region in the parameter space where the phase transition must occur. The inset shows the result of a large number of molecular dynamics simulations, performed in the close vicinity of the phase transition. The abrupt change in magnetization, as $u$ is varied indicates a first-order phase transition, as is predicted by the theory. For all simulations, the integration was performed up to $t=1200$. The final $M_{s}$ was obtained by averaging the magnetization over an additional time interval $\Delta t=800$. We note that the theory based on Lynden-Bell's coarse-grained entropy incorrectly predicts that the phase transition at this point will be of second order [8]. 


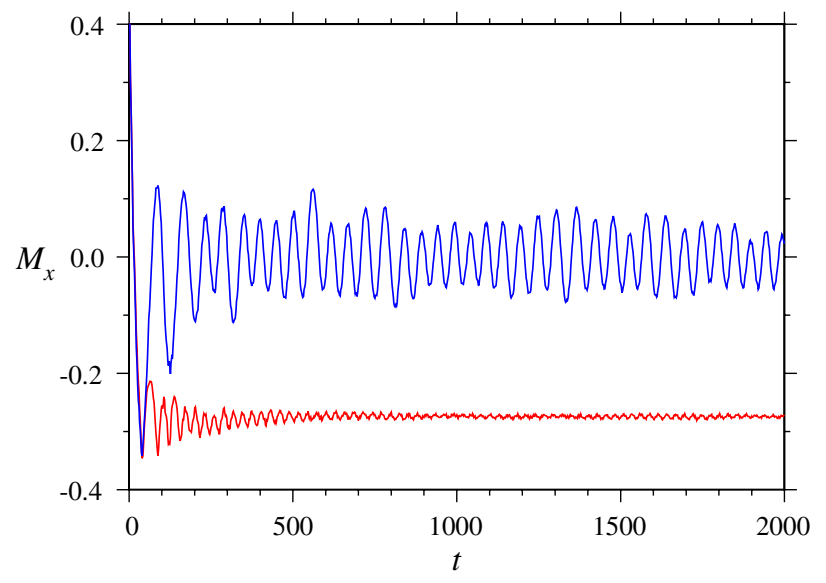

FIG. 3 (color online). Dynamical evolution of magnetization on two sides of the first-order phase transition. On the paramagnetic side $(u=0.6170)$, the magnetization oscillates around zero, while on the ferromagnetic side $(u=0.6165)$, the oscillations are damped and magnetization converges to $M_{s}$ predicted by the theory.

final stationary state is $M_{s}=0.56$. In panels (c) and (d) we compare the theoretically calculated spin and momentum distributions with the molecular dynamics simulations. As can be seen, excellent agreement is found between the theory and the simulations.

For all the initial distributions with finite magnetization, we find that if the energy per particle is less than $u<u_{l}$ there are two real roots of Eq. (8), $M_{s}=0$ and $M_{s} \neq 0$. The root $M_{s}=0$ is unstable, so that only the solution with finite magnetization has a physical meaning. On the other hand for $u>u_{u}$, there is only one real root, $M_{s}=0$. For the interval of energies $u_{l} \leq u \leq u_{u}$ there are three distinct roots, see Fig. 2. The theory, therefore, predicts that there is a first-order phase transition in the interval between $u_{l} \leq$ $u \leq u_{u}$. This is precisely what is found in simulations, see Fig. 2. Unfortunately, differently from the equilibrium phase transitions, here we do not have a free energy, which would allow us to precisely locate the transition point using the Maxwell construction. All we can do is delimit the location of the first-order phase transition within a narrow interval $u_{l} \leq u \leq u_{u}$, shaded gray in Fig. 2. On the paramagnetic side of the phase transition, the systems becomes trapped in an out-of-equilibrium limit cycle associated with appearance of resonance islands in the phase space [13], characterized by strong oscillation of magnetization around $M=0$, see Fig. 3 .

We have studied the paradigmatic system with longrange interactions, the so-called Hamiltonian mean-field model. It is shown that in the thermodynamic limit this model does not relax to the usual Maxwell-Boltzmann distribution. Instead the final stationary state of the HMF has a core-halo distribution function which is an ansatz solution to the Vlasov equation. The theory allows us to explicitly calculate the spin and the momentum distribution functions, both of which are found to be in excellent agreement with the simulations, without any adjustable parameters. We also find that the HMF model undergoes a first-order ferro-to-para phase transition in the region of parameter space where earlier theories based on the Lynden-Bell (LB) coarse-grained entropy predicted a second order phase transition [8]. It is interesting to note that the previous simulations $[8,14]$ - performed over a fairly short time span $t<150$, before the system has fully relaxed-failed to notice this incorrect prediction of the LB theory.

The present theory - as well as the earlier results on nonneutral plasmas [2], and self-gravitating systems in one [15] and two [3] dimensions-suggests that there is a significant degree of universality in collisionless relaxation dynamics. The core-halo distribution function appears to be a universal attractor-in a coarse-grained sense-of systems with long-range interactions, analogous to the MB distribution for collisional systems with short-range forces.

This work was partially supported by the CNPq, FAPERGS, INCT-FCx, and by the US-AFOSR under Grant No. FA9550-09-1-0283.

[1] J. W. Gibbs, Collected Works (Longmans, Green and Co., New York, 1928).

[2] Y. Levin, R. Pakter, and T. N. Teles, Phys. Rev. Lett. 100, 040604 (2008).

[3] T. N. Teles, Y. Levin, R. Pakter, and F. B. Rizzato, J. Stat. Mech. (2010) P05007.

[4] K. Jain et al., J. Stat. Mech. (2007) P11008.

[5] J. Barré, D. Mukamel, and S. Ruffo, Phys. Rev. Lett. 87, 030601 (2001).

[6] D. Mukamel, S. Ruffo, and N. Schreiber, Phys. Rev. Lett. 95, 240604 (2005).

[7] A. Campa, T. Dauxois, and S. Ruffo, Phys. Rep. 480, 57 (2009).

[8] A. Antoniazzi, D. Fanelli, S. Ruffo, and Y. Y. Yamaguchi, Phys. Rev. Lett. 99, 040601 (2007).

[9] A. Antoniazzi, D. Fanelli, J. Barré, P. H. Chavanis, T. Dauxois, and S. Ruffo, Phys. Rev. E 75, 011112 (2007).

[10] A. Antoniazzi, F. Califano, D. Fanelli, and S. Ruffo, Phys. Rev. Lett. 98, . 150602 (2007); J. Barre, F. Bouchet, T. Dauxois, and S. Ruffo, Phys. Rev. Lett. 89, 110601 (2002); T. M. Rocha Filho, A. Figueiredo, and M. A. Amato, Phys. Rev. Lett. 95, 190601 (2005).

[11] W. Braun and K. Hepp, Commun. Math. Phys. 56, 101 (1977).

[12] L. Landau, J. Phys. USSR 10, 25 (1946).

[13] R. Bachelard, C. Chandre,D. Fanelli, X. Leoncini, and S. Ruffo, Phys. Rev. Lett. 101, 260603 (2008).

[14] F. Staniscia, P. H. Chavanis, G. De Ninno, and D. Fanelli, Phys. Rev. E 80, 021138 (2009).

[15] M. Joyce and T. Worrakitpoonpon, arXiv:1012.5042.

[16] T. N. Teles, R. Pakter, and Y. Levin, Appl. Phys. Lett. 95, 173501 (2009). 